\title{
A new mechanism of mass protection for fermions*
}

\author{
J.L. Alonso ${ }^{\text {a }}$, Ph. Boucaud ${ }^{b}$, J.M. Carmona ${ }^{\text {, }}$ J.L. Cortés ${ }^{\mathrm{a}}$, J. Polonyi ${ }^{\mathrm{d}}$, A.J. van der Sijs ${ }^{\mathrm{e}}$ \\ ${ }^{a}$ Departamento de Física Teórica, Facultad de Ciencias, Univ. de Zaragoza, 50009 Zaragoza, Spain \\ ${ }^{\text {b} L a b o r a t o i r e ~ d e ~ P h y s i q u e ~ T h e ́ o r i q u e ~ e t ~ H a u t e s ~ E ́ n e r g i e s, ~ U n i v . ~ d e ~ P a r i s ~ X I, ~} 91405$ Orsay, France \\ ${ }^{\mathrm{c}}$ Dipartimento di Fisica, Università di Pisa, Via Buonarroti, 2, 56127 Pisa, Italy \\ ${ }^{\mathrm{d}}$ Laboratoire de Physique Théorique, Univ. Louis Pasteur, 67084 Strasbourg Cedex, France, and \\ Department of Atomic Physics, L. Eötvös University, Budapest, Hungary \\ ${ }^{\text {eSwiss }}$ Center for Scientific Computing, ETH Zürich, ETH-Zentrum, CH-8092 Zürich, Switzerland
}

\begin{abstract}
We present a way of protecting a Dirac fermion interacting with a scalar (Higgs) field from getting a mass from the vacuum. It is obtained through an implementation of translational symmetry when the theory is formulated with a momentum cutoff, which forbids the usual Yukawa term. We consider that this mechanism can help to understand the smallness of neutrino masses without a tuning of the Yukawa coupling. The prohibition of the Yukawa term for the neutrino forbids at the same time a gauge coupling between the right-handed electron and neutrino. We prove that this mechanism can be implemented on the lattice.
\end{abstract}

\section{HIGGS MECHANISM (SM)}

The Higgs mechanism is the mechanism to give mass to fermions and gauge bosons in the Standard Model (SM). However, in the SM there are massless fermions: the neutrinos. In fact, a righthanded neutrino $\nu_{R}$ is not introduced so that it remains massless. However, most of the extensions of the SM imply the existence of a $\nu_{R}$. With the introduction of a $\nu_{R}$, the neutrino can be coupled to the Higgs field and get a Dirac mass $m_{\nu_{e}}\left(\bar{\nu}_{e_{L}} \nu_{e_{R}}+\right.$ h.c. $)$. A fundamental problem is then to understand why $m_{\nu_{e}} / m_{e}$ is such a small number $\left(<10^{-5}\right)$. In the following we will give a possible answer to this problem by means of a mechanism to protect a fermion coupled to the Higgs field from acquiring a mass from the Higgs vacuum.

\section{MASS PROTECTION MECHANISM}

We present the following mechanism, based on two characteristics of the SM: first, the freedom

\footnotetext{
*Presented by J. M. Carmona (carmona@difi.unipi.it). Partially supported by CICyT (Spain) AEN97-1680, AEN97-1708, and TMR Network no. FMRX-CT97-0122.
}

in the choice of the representations of the symmetries of the theory in which the elementary particles appear (we will consider specifically the translational symmetry) and second, that it is a low-energy effective theory. This last fact implies the presence of a momentum cutoff scale $\Lambda$. We will identify new representations of the translational symmetry considering that the momentum cutoff $-\Lambda \leq p_{\mu} \leq \Lambda$ naturally reduces the Poincaré group of symmetry to a discrete subgroup: in Euclidean space, that generated by rotations of $\pi / 2$ in each plane, and by translations of $\pi / \Lambda$ in each direction.

There are only two different representations of discretized translations $\left(r^{\mu}=n^{\mu} \pi / \Lambda, n^{\mu} \in \mathbb{Z}\right)$ which are compatible with the usual representations of rotations:

$\psi^{\prime}(p)=e^{i r \cdot p} \psi(p) \quad, \quad$ and
$\psi^{\prime}(p)=e^{i r \cdot \tilde{p}} \psi(p) \quad, \quad \tilde{p}_{\mu}=p_{\mu}-\Lambda \operatorname{sign}\left(p_{\mu}\right)$

In coordinate space, these representations are:

$\psi^{\prime}(x)=\psi(x+r)$
$\psi^{\prime}(x)=e^{i \Lambda \sum_{\mu} r_{\mu}} \psi(x+r)$

To illustrate how the mass protection mecha- 
nism works, we will consider a chiral model with a left and a right fermion coupled to a complex scalar field, and a different representation under translations for each chirality of the fermion field. The physical interpretation of this will be that the two chiralities are coupled differently to the physics beyond the cutoff. Then, the usual Yukawa term in momentum space,

$y \bar{\psi}_{L}([p+k]) \phi(k) \psi_{R}(p)$,

is forbidden by translational invariance. In Eq. (5), $[p+k]$ is the momentum compatible with the cutoff obtained by adding or substracting if necessary $2 \Lambda$ to the components of $p+k$. The interaction term compatible with the new implementation of translations is

$y \bar{\psi}_{L}(\widetilde{[p+k]}) \phi(k) \psi_{R}(p)$,

where the tilde symbol was already introduced in Eq. (2). At leading order for the fermion propagator, one finds, in the case of the term (5), a free fermion with mass $m=y\langle\phi\rangle$, and in the case of the term (6), a massless fermion up to corrections proportional to inverse powers of the cutoff $\Lambda$.

However, as the term (6) couples momentum modes that differ in $\Lambda$, a nonperturbative implementation of this mechanism could be problematic owing to the well-known fermion doubling phenomenon. Let us see that this is not the case.

\section{LATTICE IMPLEMENTATION}

On the lattice, we take for the representation of translations for the fermion field :

$\psi_{L x}^{\prime}=e^{i \alpha_{L}} \psi_{L x+\hat{\mu}}, \quad \psi_{R x}^{\prime}=e^{i \alpha_{R}} \psi_{R x+\hat{\mu}}$,

under a translation of one lattice spacing in the $\hat{\mu}$ direction. As in the previous discussion, we will take $\alpha_{L}=0$ and $\alpha_{R}=\pi$ in order to have compatibility with the usual representations of rotations.

The translational invariant lattice action is

$S=S_{\mathrm{B}}+S_{\mathrm{F}}+S_{\mathrm{FB}}$

where

$$
S_{\mathrm{B}}=-\kappa \sum_{x, \mu}\left(\phi_{x}^{*} \phi_{x+\hat{\mu}}+\phi_{x+\hat{\mu}}^{*} \phi_{x}\right)
$$

$$
\begin{aligned}
& +\sum_{x}\left\{\phi_{x}^{*} \phi_{x}+\lambda\left(\phi_{x}^{*} \phi_{x}-1\right)^{2}\right\}, \\
S_{\mathrm{F}}= & \sum_{x, \mu} \frac{1}{2}\left(\bar{\psi}_{x} \gamma_{\mu} \psi_{x+\hat{\mu}}-\bar{\psi}_{x+\hat{\mu}} \gamma_{\mu} \psi_{x}\right), \\
S_{\mathrm{FB}}= & y \sum_{x}(-1)^{\sum_{\nu} x_{\nu}}\left(\bar{\psi}_{L x}^{(1)} \phi_{x} \psi_{R x}^{(1)}\right. \\
& \left.+\bar{\psi}_{R x}^{(1)} \phi_{x}^{*} \psi_{L x}^{(1)}\right),
\end{aligned}
$$

with

$\psi^{(1)}(p)=F(p) \psi(p)$.

$F(p)$ is a form factor required to be 1 for $p=0$ and to vanish when $p$ equals any of the doubler momenta. With this method, we have a theory with 16 fermions, 15 of which do not interact with physical particles and decouple from the real world 11.

In order to do perturbation theory, let us set

$\phi_{x}=\phi_{1 x}+i \phi_{2 x}$,

and consider a scalar field $\phi$ with a $\operatorname{VEV}\left\langle\phi_{1 x}\right\rangle=$ $v,\left\langle\phi_{2 x}\right\rangle=0$. Then we write

$\phi_{1 x}=v+\eta_{1 x}, \quad \phi_{2 x}=\eta_{2 x}$,

where $\eta_{1,2}$ represent the small perturbations.

Let us first note that the presence in the action of the term $S_{\mathrm{FB}}$ with such an unusual coupling does not modify the vacuum $\left\langle\phi_{1 x}\right\rangle=v$. This is a consequence of both analytical and numerical studies of the antiferromagnetic (AFM) phase of the chiral Yukawa model [2]. Under the change of variables $\phi_{x}^{\prime}=\varepsilon_{x} \phi_{x}$, where $\varepsilon_{x}=(-1)^{\sum_{\nu} x_{\nu}}$, the action is invariant if the couplings are mapped according to

$\left(\kappa, y \varepsilon_{x}\right) \longmapsto(-\kappa, y)$.

With these couplings, a stable AFM phase exists where the scalar gets a staggered mean value $\left\langle\phi_{1 x}^{\prime}\right\rangle=\varepsilon_{x} v_{\text {st }}$. We can then conclude that the original vacuum $\left\langle\phi_{1 x}\right\rangle=v$ is also a stable vacuum for the action (8).

In momentum space, the inverse of the fermion propagator at tree-level order is

$i \delta\left(p-p^{\prime}\right) \not(p)+y v F(p) F_{\pi}(p) \delta\left(p-p^{\prime}+\boldsymbol{\pi}\right)$,

where $\$(p)=\sum_{\mu} \gamma_{\mu} \sin p_{\mu}, F_{\pi}(p) \equiv F(p+\pi)$, and $\boldsymbol{\pi} \equiv(\pi, \pi, \pi, \pi)$. We have $F_{\pi}(0)=0$. This 
matrix is not diagonal in momentum space, as it connects $p$ with $p+\boldsymbol{\pi}$ in a box of the form

$G^{-1}(p)=\left(\begin{array}{cc}i \not(p) & y v F(p) F_{\pi}(p) \\ y v F_{\pi}(p) F(p) & i \not(p+\pi)\end{array}\right)$

It can be diagonalized to give

$G_{\mathrm{D}}=\frac{1}{i \not(p)+i m(p)}, m(p)=y v F(p) F_{\pi}(p)$.

This propagator has 16 poles at momenta $(0,0,0,0),(\pi, \pi, \pi, \pi),(\pi, 0,0,0),(\pi, \pi, 0,0)$, etc., which implies zero mass at tree level for the physical fermion and all the doublers. One can see through perturbative calculations and nonperturbative arguments that this masslessness is maintained at every loop order [3].

We are interested in the continuum limit of the theory because we want to apply it to energy scales $E \ll \Lambda$. In the limit $\Lambda \rightarrow \infty$, the propagator (18) becomes $(-i) / p$, that is, a massless fermion propagator. This limit is well defined because it corresponds to the second order phase transition of the AFM phase in the chiral Yukawa model, where we have restoration of rotational invariance and renormalizability of the theory. In summary, we have obtained a massless fermion in the low-energy theory by using transformation laws under the symmetries of the theory related to the presence of the scale $\Lambda$, that is, related to the properties of the theory at the next level $E>\Lambda$.

\section{APPLICATION TO THE SM}

The present mechanism could be applied in the framework of the SM with $\nu_{R}$ to understand the absence of a neutrino Dirac mass, by simply choosing a different representation for the $\mathrm{L}$ and $\mathrm{R}$ chiralities of this fermion under translational symmetry. As in the $\mathrm{SM} e_{L}$ and $\nu_{L}$ are coupled by the gauge field, they should appear in the same representation, together with $e_{R}$ (in order to have the usual Higgs mechanism for the electron). Then the right-handed electron and the right-handed neutrino are in different representations and they cannot be in the same weak isospin multiplet. This situation is in fact assumed in the SM.
Recent oscillation results [4] suggest that the neutrino could have a small mass. The usual explanation for this requires the introduction of Majorana terms, which violate lepton number conservation. In the framework of the minimal SM as an effective theory, a Majorana mass can be generated for the neutrino by the dimension five operator

$\psi_{L}^{\mathrm{T}}(x) C \psi_{L}(x) \phi(x) \phi(x)$.

In the SM with $\nu_{R}$, the see-saw mechanism [5] balances the Dirac term $\bar{\psi}_{L}(x) \psi_{R}(x)$ and the Majorana term $\psi_{R}^{\mathrm{T}}(x) C \psi_{R}(x)$ in order to explain a small mass for the neutrino.

The mass protection mechanism proposed in this work allows a $\nu_{R}$ in the theory without the generation of a Dirac mass. Also, a scenario of almost-degenerate neutrinos (the relevant one in cosmology) could be explained in an easier way after having eliminated the hierarchy of the Dirac mass matrix. Besides that, if no Majorana terms are allowed in the model, the neutrino oscillations could be due to effects of order $v / \Lambda$, and compatible with lepton number conservation in the framework of this mass protection mechanism.

\section{REFERENCES}

1. J.L. Alonso, Ph. Boucaud, J.L. Cortés and E. Rivas, Phys. Rev. D 44, 3258 (1991); J.L. Alonso, Ph. Boucaud, F. Lesmes and A.J. van der Sijs, Nucl. Phys. B 457, 175 (1995).

2. W. Bock, A.K. De, K. Jansen, J. Jersák, T. Neuhaus and J. Smit, Nucl. Phys. B 344, 207 (1990).

3. J.L. Alonso, Ph. Boucaud, J.M. Carmona, J.L. Cortés, J. Polonyi and A.J. van der Sijs, hep-th/9807181.

4. C. Athanassopoulos et al., Phys. Rev. Lett. 77, 3082 (1996); Y. Fukuda et al., Phys. Rev. Lett. 82, 2644 (1999).

5. T. Yanagida, in Proc. of Workshop on Unified Theory and Baryon Number of the Universe, eds. O. Sawada and A. Sugamoto (KEK, 1979); M. GellMann, P. Ramond and R. Slanksky, in Supergravity, eds. P. von Nienvenhuizen and D.Z. Freedman (N. Holland, 1979). 